\title{
Progress towards ultra sensitive KIDs for future far-infrared missions: a focus on recombination times
}

Adalyn Fyhrie, Jonas Zmuidzinas, Jason Glenn, Peter Day, Henry G. LeDuc, et al.

Adalyn Fyhrie, Jonas Zmuidzinas, Jason Glenn, Peter Day, Henry G. LeDuc, Christopher McKenney, "Progress towards ultra sensitive KIDs for future farinfrared missions: a focus on recombination times," Proc. SPIE 10708, Millimeter, Submillimeter, and Far-Infrared Detectors and Instrumentation for Astronomy IX, 107083A (24 August 2018); doi: 10.1117/12.2312867

Event: SPIE Astronomical Telescopes + Instrumentation, 2018, Austin, Texas, United States 


\title{
Progress Towards Ultra-Sensitive KIDs for Future Far-Infrared Missions: A Focus on Recombination Times
}

\author{
Adalyn Fyhrie ${ }^{\mathrm{a}}$, Jonas Zmuidzinas ${ }^{\mathrm{b}}$, Jason Glenn ${ }^{\mathrm{a}}$, Peter Day ${ }^{\mathrm{c}}$, Henry G. LeDuc ${ }^{\mathrm{c}}$, and \\ Christopher McKenney ${ }^{\mathrm{d}}$ \\ a University of Colorado Boulder, 2000 Colorado Avenue, Boulder, USA \\ ${ }^{\mathrm{b}}$ California Institute of Technology, 1200 East California Boulevard, Pasadena, USA \\ c Jet Propulsion Laboratory, 4800 Oak Grove Dr, Pasadena, USA \\ dNational Institute of Standards and Technology, 325 Broadway, Boulder, USA
}

\begin{abstract}
Future generations of far-infrared (FIR) telescopes will need detectors with noise-equivalent powers on the order of $5 \times 10^{-20} \mathrm{~W} / \mathrm{Hz}^{1 / 2}$ in order to be photon background limited by astrophysical sources. One such mission concept in development is the Galaxy Evolution Probe (GEP), which will characterize galaxy formation and evolution from $\mathrm{z}=0$ to beyond $\mathrm{z}=4$. Kinetic inductance detectors (KIDs) have been baselined for the GEP for spectroscopy and imaging science between $10 \mu \mathrm{m}$ and $400 \mu \mathrm{m}$ due to their intrinsic frequency multiplexability and simple readout schemes. We focus on quasiparticle recombination times as a strategy for increasing detector responsivities to move towards the NEP requirements of the GEP. We present a new model for quantifying time constants from the responses of detectors to pulses of light, and test this model on a $40 \mathrm{~nm}$ thick $1 / 4$ $\lambda \mathrm{Al}$ coplanar waveguide KID. We intend to use this measurement scheme to quantify the dependence of the quasiparticle recombination time on $\mathrm{Al}$ thickness.
\end{abstract}

Keywords: Kinetic Inductance Detectors, Far-Infrared, Galaxy Evolution Probe, Quasiparticle Recombination

\section{INTRODUCTION}

Infrared astronomers have enjoyed the use of extremely powerful telescopes over the last few decades. Spacebased observatories like Herschel Space Observatory expanded our understanding of galaxy evolution and star formation on Galactic and universal scales. Ground-based observatories such as ALMA are providing insight into galactic physics and evolution with its capability for high-resolution, detailed observations of nearby galaxies.

With Herschel's retirement, a telescope that can carry out large surveys in the mid- and far-infrared is needed. James Webb Space Telescope (JWST) will do this job well at wavelengths below $30 \mu \mathrm{m}$ and ALMA excels at detailed observations above $300 \mu \mathrm{m}$. However, the intermediate wavelengths needed for dust emission from local galaxies and redshifted rest-frame mid-infrared emission from galaxies at higher redshifts, as well as the ability to survey large swaths of sky and discover new objects, will be critical to develop our understanding of star formation and galaxy evolution over time.

One option that suits this goal is the Galaxy Evolution Probe (GEP), ${ }^{1}$ a space observatory budgeted at under $\$ 1 \mathrm{~B}$ developed as part of NASA's Astrophysics Probe concept development program. The GEP is designed to survey star-forming galaxies from $\mathrm{z}=0$ to greater than $\mathrm{z}=4$ using spectroscopy and broadband imaging in wavelength bands from 10-400 $\mu \mathrm{m}$. Its current design goal is to have sensitivities limited by photon noise from astrophysical backgrounds and not by emission from the telescope itself, which will be cryogenically cooled to 4 $\mathrm{K}$ to reduce infrared emission. This would require large arrays of detectors with noise equivalent powers (NEPs) on the order of $10^{-18} \mathrm{~W} / \mathrm{Hz}^{1 / 2}$ for the imager and $10^{-19} \mathrm{~W} / \mathrm{Hz}^{1 / 2}$ for the spectrometer.

Further author information: (Send correspondence to Adalyn Fyhrie)

Adalyn Fyhrie: E-mail: adalyn.fyhrie@colorado.edu

Millimeter, Submillimeter, and Far-Infrared Detectors and Instrumentation for Astronomy IX, edited by Jonas Zmuidzinas, Jian-Rong Gao, Proc. of SPIE Vol. 10708, 107083A

(C) 2018 SPIE · CCC code: 0277-786X/18/\$18 · doi: 10.1117/12.2312867

Proc. of SPIE Vol. 10708 107083A-1 
Kinetic inductance detectors (KIDs) have been baselined as the detectors for the GEP due to their simple focal plane architecture as compared to transition edge sensors (TESs). TESs and their readout technologies, however, have higher TRLs than KIDs and therefore are a viable option depending on the state of each technology during future design studies.

The development of KID technologies has become a worldwide endeavor, with many promising NEPs resulting from these efforts: Janssen et al. $2014^{2}$ achieved an NEP of $1.4 \times 10^{-18} \mathrm{~W} / \mathrm{Hz}^{1 / 2}$ at $350 \mu \mathrm{m}$ for optical loading of $0.1 \mathrm{fW}$, and P. Diener et al. ${ }^{3}$ reached an array-averaged dark NEP of $5.4 \times 10^{-19} \mathrm{~W} / \mathrm{Hz}^{1 / 2}$, with some pixels as low as $4.4 \times 10^{-20} \mathrm{~W} / \mathrm{Hz}^{1 / 2}$. For TESs, derived NEPs as low as $7 \times 10^{-20} \mathrm{~W} / \sqrt{H z}$ have been achieved in the Far-IR. ${ }^{4}$ These NEPs are approaching the array-scale low NEPs needed by the GEP, but further KID development is needed to produce large arrays of low-NEP detectors at the wavelengths needed for the GEP.

In this paper, we focus on increasing the responsivity of low-volume aluminum KIDs as a step towards low NEPs, with an architecture that is extensible to shorter wavelengths. We do this in two ways: first, minimize the detector's active volume, to which the responsivity is inversely proportional. Second, maximize the quasiparticle recombination time $\tau_{q p}$ (Section 3.2), to which the responsivity is proportional. To find an optimal geometry that minimizes detector volume while maintaining long $\tau_{q p}$ 's, we seek to quantify the dependence of $\tau_{q p}$ on $\mathrm{Al}$ film thickness. To measure $\tau_{q p}$, we present a new model for measuring quasiparticle recombination times in detectors. We test this model on data from a $40 \mathrm{~nm}$ thick $1 / 4 \lambda \mathrm{Al}$ coplanar waveguide (CPW) KID array.

\section{APPROACH FOR MAXIMIZING KINETIC INDUCTANCE DETECTOR RESPONSIVITIES}

In order to maximize $\tau_{q p}$ while minimizing detector volume, we seek to understand the influence of a detector's metal thickness on the recombination time. For this test, we use devices made of aluminum because time constants as long as a millisecond have been observed in aluminum in the past ${ }^{5,6}$ (thicknesses of $250 \mathrm{~nm}$ and 40 $\mathrm{nm}$ on sapphire, respectively). However, the minimum $\mathrm{Al}$ thickness required to achieve these lifetimes has not been quantified directly.

To minimize detector volume, the ideal design is to have extremely thin lines, but our previous measurements of time constants for thin $(20 \mathrm{~nm})$ Al LEKID devices revealed extremely short time constants of $60 \mu \mathrm{s}^{7}$. We suspect that the short time constants were due to the layer of aluminum oxide that forms on all $\mathrm{Al}$ devices and degrades both resonator quality factors and quasiparticle recombination times. The characteristic thickness of this oxide layer is $4 \mathrm{~nm}$ regardless of metal thickness, ${ }^{8}$ so its influence on resonator performance may decrease as the metal is thickened.

To understand how much thicker than $20 \mathrm{~nm}$ the $\mathrm{Al}$ must become to achieve the desired long $\tau_{q p}$ 's of around $1 \mathrm{~ms}$, we fabricated pure $\mathrm{Al} 1 / 4 \lambda$ coplanar waveguide (CPW) test arrays with thicknesses varying from $10 \mathrm{~nm}$ to $100 \mathrm{~nm}( \pm 10 \%)$ and will measure the recombination time for each. Future iterations of KID arrays will be designed with the minimum thickness required for long $\tau_{q p}$ 's.

\section{MEASURING QUASIPARTICLE RECOMBINATION TIMES}

\subsection{Test Setup}

To measure $\tau_{q p}$, we deliver a pulse of infrared light through an optical fiber to a KID array mounted in an otherwise light-tight box attached to an adiabatic dilution refrigerator. The optical fiber is strung from $300 \mathrm{~K}$ to the detector stage and heat sunk on the $4 \mathrm{~K}$ and $1 \mathrm{~K}$ stages. The devices are not coupled to the light by lenses or feedhorns, and the optical fiber is offset by approximately 2.5 inches from the array. Therefore, we assume uniform illumination of the array and the ground plane. This should prevent the diffusion of quasiparticles out of the detectors into the surrounding ground plane from affecting our measurements of $\tau_{q p}$.

The infrared light is produced by an LED (Thorlabs LED1200L) with a peak wavelength of $1200 \mathrm{~nm}$ and a FWHM of $70 \mathrm{~nm}$. This wavelength was chosen to avoid producing photoelectrons in the silicon on which the aluminum layer is deposited. These photoelectrons, excited by light with $\lambda<1100 \mathrm{~nm}$, can affect measurements of $\tau_{q p}$ due to their own intrinsic timeconstant after which they recombine with a hole and produce phonons. 
The LED is controlled with a function generator that turns the LED on for $4 \mathrm{~ms}$ at a time every 0.25 seconds. We collect 45 seconds of data, meaning that each $\tau_{q p}$ measurement is made on an average detector response comprised of 180 individual pulses.

During these tests, we had optical windows installed on the $4 \mathrm{~K}, 50 \mathrm{~K}$, and $300 \mathrm{~K}$ shields of the cryostat, while the $1 \mathrm{~K}$ and $100 \mathrm{mK}$ stages were designed to be light-tight. We tested the effectiveness of our lighttight boxes by by placing sources of different reflectivities and temperatures in front of the $300 \mathrm{~K}$ window and observing the detector resonances. We observed resonance frequency shifts up to two line widths when placing room-temperature sources in front of the $300 \mathrm{~K}$ window, indicating that our system was not light-tight. This means that all measurements presented for $\tau_{q p}$ are for optically loaded devices, indicating that they may be lower bounds.

\subsection{Quasiparticle Recombination}

\subsubsection{Motivation}

When a superconductor absorbs radiation, the superconducting charge carriers, called Cooper pairs, break into individual electrons deemed "quasiparticles". These quasiparticles then recombine after an average time $\tau_{q p}$, called the quasiparticle recombination time. If a pulse of light (in our case produced by an LED) is absorbed by a KID, the expected signal after the LED turns off is an exponential decay to zero of fractional frequency shift $(\Delta f / f)$ over time of the form $e^{-t / \tau_{q p}}$. However, this is complicated by the fact that the rate of quasiparticle recombination is proportional to quasiparticle density. High densities of quasiparticles result in faster recombination times because quasiparticles are able to find a partner to recombine with more easily, and the inverse is true for lower densities of quasiparticles. Consequently, as quasiparticles recombine after a pulse of light, the quasiparticle density naturally decreases and hence the signal can appear to decay more quickly at first before it slows due to the lower quasiparticle density. This can result in a deviation from a single exponential decay of $\Delta f / f$ over time.

Our measurements showed a deviation from a single exponential (see Figure 1), showing instead fast and then slow decay, motivating the creation of a new mathematical model for fitting for $\tau_{q p}$.

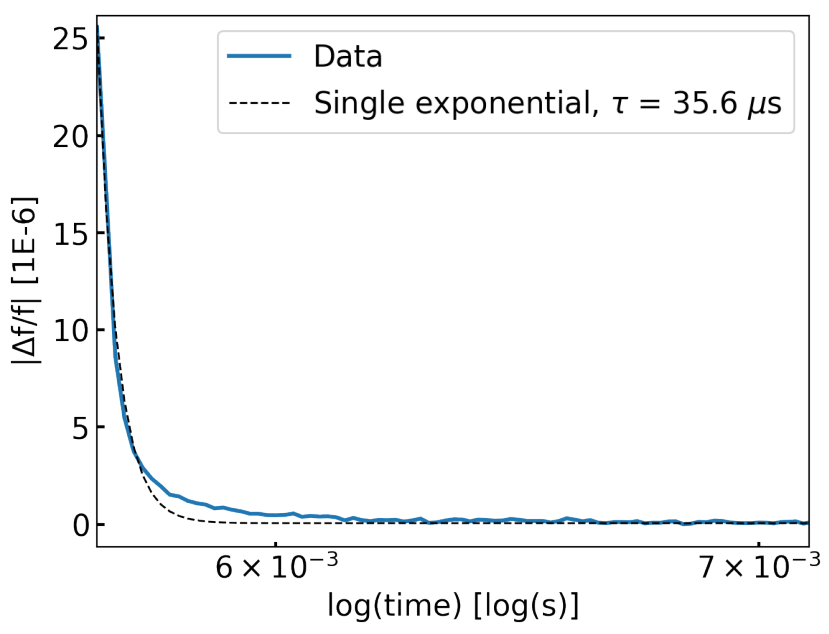

Figure 1. Data (blue) showing a typical decay of $\Delta f / f$ vs time for a detector at $100 \mathrm{mK}$, and a single exponential fit (dashed black). The exponential captures the quick decay at the beginning of the pulse, but fails to capture the slower decay at the tail end. 


\subsubsection{Mathematical Model for Non-Exponential Decay}

To characterize decays in fractional frequency shift $(\Delta f / f)$ in KIDs that absorb pulses of light, we begin with the rate equation governing quasiparticle recombination:

$$
\frac{d N_{q p}(t)}{d t}=-\gamma_{r} N_{q p}(t)+I_{q p}(t),
$$

where $N_{q p}(t)$ is the number of quasiparticles with time, $\gamma_{r}$ is the recombination rate, and $I_{q p}(t)$ is the quasiparticle generation rate with time.

The recombination rate $\gamma_{r}$, as mentioned previously, increases with the number of quasiparticles. Additionally, experiments show that it is never zero - rather, it decreases to some fiducial value at a characteristic quasiparticle density. This behavior is similar to that of $\tau_{q p}$ observed in experiments, ${ }^{5}$ which follows the form

$$
\tau_{q p}=\frac{\tau_{\max }}{1+n_{q p} / n_{*}} .
$$

Here, $n_{q p}$ is the thermal quasiparticle density and $n_{*}$ is a fiducial density below which $\tau_{q p}=\tau_{\max }$. Using this observed behavior of $\tau_{q p}$, we can write

$$
\gamma_{r}=\frac{1}{\tau_{\max }}\left(1+\frac{n_{q p}}{2 n_{*}}\right) .
$$

The factor of 2 in the denominator is introduced for consistent definitions of $n_{*}$ between Equations 2 and 3 . It can be found by combining Equations 3 and 1 and integrating for a small perturbation $\Delta N$ to a steady-state quasiparticle population of $N$.

Noting that $n_{q p} / n_{*}=N_{q p} / N_{*}$ where $n_{q p}$ and $n_{*}$ are densities in a volume $\mathrm{V}$ and $N_{q p}$ and $N_{*}$ are total numbers in a volume $\mathrm{V}$, we can combine Equations 3 and 1 to write

$$
\frac{d N_{q p}(t)}{d t}=-\frac{1}{\tau_{\max }}\left[1+\frac{N_{q p}(t)}{2 N_{*}}\right] N_{q p}(t)+I_{q p}(t) .
$$

In our testing scenario, we deliver a quick pulse of light to a test device and then return to a dark state, meaning $\mathrm{I}_{q p}=0$ during the decay of $\Delta f / f$ back to zero. By setting $\mathrm{I}_{q p}=0$ in Equation 4 and by introducing the variable $x_{q p}=N_{q p}(t) / 2 N_{*}$ for ease of calculation, we come to our final governing rate equation of

$$
\frac{d x_{q p}(t)}{d t}=-\frac{1}{\tau_{\max }}\left[1+x_{q p}(t)\right] x_{q p}(t) .
$$

By integrating and rearranging terms, we can write the equation that governs $\mathrm{x}_{q p}(\mathrm{t})$ as

$$
x_{q p}(t)=\frac{1}{\left[1+1 / x_{q p}(0)\right] \exp \left(t / \tau_{\max }\right)-1} .
$$

The physical meaning of the initial value $\mathrm{x}_{q p}(0)$ that is a byproduct of integration can be verified by examining the limits of Equation 6 for $\mathrm{x}_{q p}(0) \ll 1$ and $\gg 1$. When $\mathrm{x}_{q p}(0) \ll 1$ we can approximate Equation 6 as an exponential decay with a characteristic decay time $\tau_{\max }$. This is the same form that results from Equation 4 for low quasiparticle density. When $\mathrm{x}_{q p}(0) \gg 1$ we can approximate Equation 6 as

$$
x_{q p}(t) \approx \frac{1}{\exp \left(t / \tau_{\max }\right)-1} .
$$

This behaves approximately as $1 / t$ for times $\mathrm{t} \ll \tau_{\max }$ and as an exponential decay for $\mathrm{t} \gg \tau_{\max }$.

Interpreting these limiting cases, it appears that our interpretation of the integration constant $\mathrm{x}_{q p}(0)$ as a measure of the starting values of quasiparticle densities is correct. For high values $(\gg 1)$, the decay will deviate from an exponential. For low values $(\ll 1)$ the decay will behave almost exactly like an exponential. Importantly, the characteristic time $\tau_{\max }$ is the same in both cases, indicating that $\tau_{\max }$ is the recombination 
time $\tau_{q p}$. Therefore, by fitting Equation 6 to the decay of signal in a KID that has absorbed a pulse of light, we can characterize the recombination time.

When fitting Equation 6 to measured data, we must convert from the physical values of $\mathrm{x}_{q p}(\mathrm{t})$ to a measured parameter $\mathrm{X}(\mathrm{t})$ (in our case $\Delta f / f$ ) which should be a linear conversion, meaning

$$
X(t)=\frac{A}{\left[1+1 / x_{q p}(0)\right] \exp \left(t / \tau_{q p}\right)-1} .
$$

However, setting $\mathrm{t}=0$ in this equation we see that $\mathrm{X}(0)=\mathrm{x}_{q p}(0) \mathrm{A}$, meaning the intial amplitude of the observed pulse constrains the product of $\mathrm{x}_{q p}(0)$ and $\mathrm{A}$ but not each independently. Our initial analysis found this equation to produce highly correlated and poorly constrained values for both $\mathrm{x}_{q p}(0)$ and $\mathrm{A}$. To mitigate the effects of the correlation, we instead define $\mathrm{B} \equiv \mathrm{x}_{q p}(0) \mathrm{A}$ and come to our final equation that we use for all fits to data

$$
X(t)=\frac{B}{\left[1+x_{q p}(0)\right] \exp \left(t / \tau_{q p}\right)-x_{q p}(0)} .
$$

We find that this reduces the correlation between the fit parameters $\mathrm{B}$ and $\mathrm{x}_{q p}(0)$ as well as the error on $\mathrm{B}$ as compared to the parameterization presented in Equation 8. Correlations between parameters are shown in Figure 2, displaying the lack of correlation between $\mathrm{x}_{q p}(0)$ and $\mathrm{B}$ as well as between $\tau_{q p}$ and $\mathrm{B}$. A correlation exists between $\tau_{q p}$ and $\mathrm{x}_{q p}(0)$, but the values of each are well constrained within their parameter spaces.
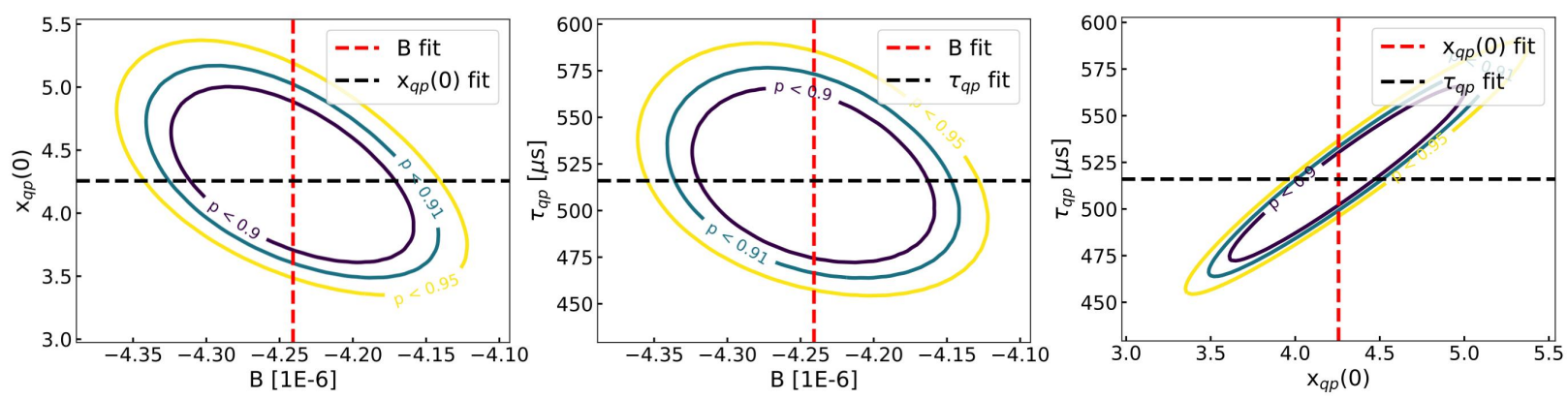

Figure 2. Results of a $\Delta \chi^{2}$ analysis for fitting Equation 9 to data from a $100 \mathrm{mK}$ resonator. $\chi^{2}$ is minimized over over $\tau_{q p}$ (left), $\mathrm{x}_{q p}(0)$ (center), and B (right). Contours show the $\Delta \chi^{2}$ values corresponding to one, two, and three $\sigma$ uncertainties in the parameters. Fit values for parameters are shown as dashed lines.

\section{RESULTS}

\subsection{Fit Parameters as a Function of Temperature}

For an array with $\mathrm{Al}$ thickness of $40 \mathrm{~nm}$, we fit Equation 9 to two detectors' decays at four temperatures of 100, 150, 200, and $250 \mathrm{mK}$. Our test system had sporadic contaminating $60 \mathrm{~Hz}$ and $60 \mathrm{~Hz}$ harmonics noise of varying amplitude that had the possibility to affect the measurement of $\tau_{q p}$. To mitigate this inconstant effect, we took many data sets for each resonator at each temperature and kept between two and three data sets at each temperature for both detectors where the noise was minimal or nonexistent. Assuming the noise's effect on the measurement of $\tau_{q p}$ is random between data sets, the average value between data sets acquired for $\tau_{q p}$ should be representative of its true value. The signal-to-noise ratio naturally decreases with increased detector temperature, and as a result the contaminating noise was too prominent to get reliable measurements above 250 $\mathrm{mK}$.

Some example fits of Equation 9 to data for both resonators are shown and discussed in Figure 3. The error bars on the data are one standard deviation $\sigma$ of the noise after the signal has completely decayed. The random scatter of the residuals around zero indicates that the model represents the data well. These fits start three data points, or $50 \mu \mathrm{s}$, after the LED turns off. The reason for this can be seen in Figure 4, which shows the beginning 
of a decay with a high signal to noise ratio, as well as the point in time at which the LED turns off. It can be seen from these data that the decay does not start immediately after the LED turns off; rather, it begins around two data points later. We saw this in the majority of our data sets with high signal to noise ratios. This is not due to the time constant of the LED nor the function generator, which both have time constants of tens of nanoseconds. We do not currently know the cause of this delay, but account for it by beginning our fits a conservative three data points after the LED is turned off.

The resultant fit parameters for both resonators as a function of temperature are shown in Figure 5. The fits and error bars are calculated using Python's curve_fit function. This function numerically calculates the Jacobian matrix $J$ of the fit function with respect to the variables $\mathrm{B}, \mathrm{x}_{q p}(0)$, and $\tau_{q p}$. It then approximates the covariance matrix $\mathrm{C}$ as $\sigma^{2} J^{T} J$, where $\sigma$ is the error calculated from the data and shown in Figure 3. The square root of the diagonals of the resultant covariance matrix are the error bars for each of our parameters. We corroborated the numerically calculated error bars with a $\Delta \chi^{2}$ fit test for the decays and found that the curve_fit errors were smaller than the one $\sigma$ uncertainties returned from the $\Delta \chi^{2}$ analysis.

The parameter $\mathrm{B}$ is determined by the height of the pulse at the beginning of the fit, which is not consistent between data sets at the same temperature. The scatter of $\mathrm{B}$ between measurements reflects this fact. $\mathrm{x}_{q p}(0)$ does not seem to change in a statistically significant way with temperature, and in many cases is not well constrained. It is reasonable to expect that $\mathrm{x}_{q p}(0)$ would increase with temperature as the number of thermal quasiparticles increases. The fact that we do not see trends with temperature for $\mathrm{x}_{q p}(0)$, as well as the large scatter between measurements, indicate that our fitting scheme is not sensitive to small changes in $\mathrm{x}_{q p}(0)$ and may need to be improved to better measure this value reliably. It is important to note, however, that even with the scatter of $\mathrm{x}_{q p}(0)$ and $\mathrm{B}$ between measurements at the same temperature, the measurements of $\tau_{q p}$ are statistically consistent with each other within $3 \sigma$ error. Additionally, the error bars on $\tau_{q p}$ are relatively small, indicating that our measurements for this crucial quantity are robust.

Equation 2 gives the expected functional form of $\tau_{q p}$ with temperature. The resultant behavior of $\tau_{q p}$ with temperature is to decrease with temperature above $\mathrm{T} / \mathrm{T}_{c} \approx 0.17$ and to stay approximately flat below that temperature at a value of $\tau_{\max }$. Our measurements of $\tau_{q p}$ in Figure 5 show, within error, the expected behavior for both resonators: an approximately flat value for lower temperatures and a steep decrease for the highest temperature $(250 \mathrm{mK})$. The turnover point of around $200 \mathrm{mK}$, which should correspond to $\mathrm{T} / \mathrm{T}_{c} \approx 0.17$, indicates that our $\mathrm{T}_{c} \approx 1.2 \mathrm{~K}$ as expected for Al. The values of $\tau_{\max }$ for each resonator are approximately 400 $\mu \mathrm{s}$ and $600 \mu \mathrm{s}$.

\section{$4.2 \mathrm{x}_{q p}(0)$ as a Function of LED Luminosity: A Test Case of Equation 9}

Given that $\mathrm{x}_{q p}(0)$ is a measure of the number of quasiparticles present at the beginning of the exponential decay, we expect its value to increase as we increase LED luminosity and, resultantly, the number of broken Cooper pairs. For a fixed detector driving power of approximately $3 \mathrm{~dB}$ below bifurcation, we varied the LED driving voltage (a proxy for luminosity) and fit Equation 9 to the resultant decay. Figure 6 shows the fit value of $\mathrm{x}_{q p}(0)$ for one resonator with aluminum thickness of $40 \mathrm{~nm}$ measured at $100 \mathrm{mK}$. As expected, $\mathrm{x}_{q p}(0)$ increases with LED driving power, indicating that our interpretation of $\mathrm{x}_{q p}(0)$ is correct.

\section{DISCUSSION AND FUTURE WORK}

Previously, our group has measured the time constants of $20 \mathrm{~nm}$ thick $\mathrm{Al}^{7}$ and $40 \mathrm{~nm}$ thick $\mathrm{Al} / \mathrm{TiN}$ bilayer LEKIDs $^{9}$ from the rolloff of the detectors' noise spectra. Both measurements yielded time constants of 60 to $100 \mu \mathrm{s}$. This was surprising for the $20 \mathrm{~nm}$ thick $\mathrm{Al}$ device because, as stated previously, time constants as long as $1 \mathrm{~ms}$ have been observed in Al-only devices. Our successful measurement of longer $\tau_{q p}>500 \mu \mathrm{s}$ is a vast improvement over these previous measurements. We speculate that the short $\tau_{q p}$ of the $20 \mathrm{~nm}$ thick Al LEKID device was due to the high proportion of aluminum oxide to un-contaminated Al. To test this, we will measure the time constants for the other Al CPW devices with thicknesses between $10 \mathrm{~nm}$ and $100 \mathrm{~nm}$.

For these future measurements, we will further light-tighten our cryostat to diminish the observed excess optical loading. We will also seek to eliminate the $60 \mathrm{~Hz}$ and $60 \mathrm{~Hz}$ harmonic contaminating noise. This should allow measurements of $\tau_{q p}$ at higher temperatures to ensure that the turnover of $\tau_{q p}$ at $\mathrm{T} \approx 0.17 \mathrm{~T}_{c} \approx 200 \mathrm{mK}$ is robust. These steps will also decrease both the scatter and error bar magnitude in our measured parameters. 

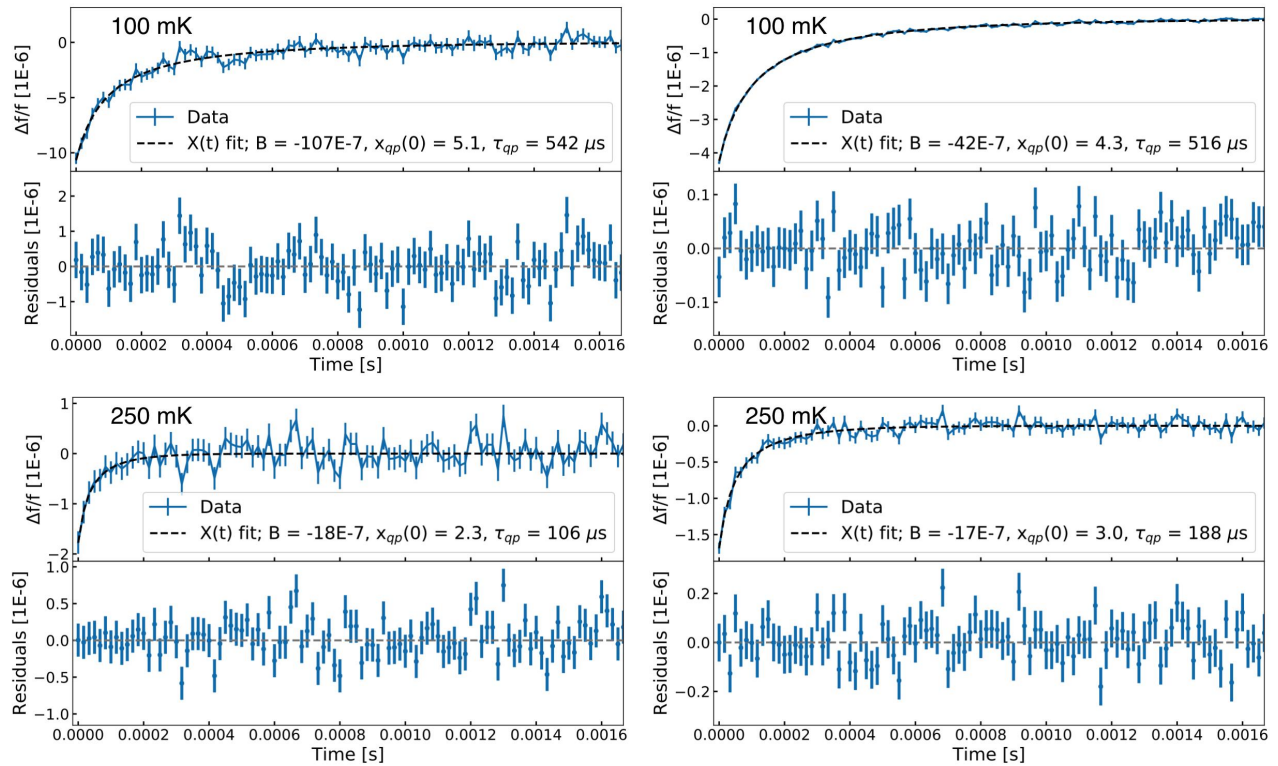

Figure 3. Example fits (black dashed lines) of Equation 9 to data (blue points and line) for two temperatures for two resonators on an aluminum device of thickness $40 \mathrm{~nm}$. The bottom sub-panels show the residuals of the fit from the data. The left column corresponds to the detector whose fit parameters are plotted together in the left column of Figure 5, and the right column corresponds to the detector whose fit parameters are plotted together in the right column of that same figure. The higher noise for the detector on the left was typical for all measurements, and likely results in the larger scatter and error bars for the fit parameters at all temperatures as compared to the other detector that are evident in Figure 5. Both resonators were driven around $3 \mathrm{~dB}$ below bifurcation with the same LED luminosity.

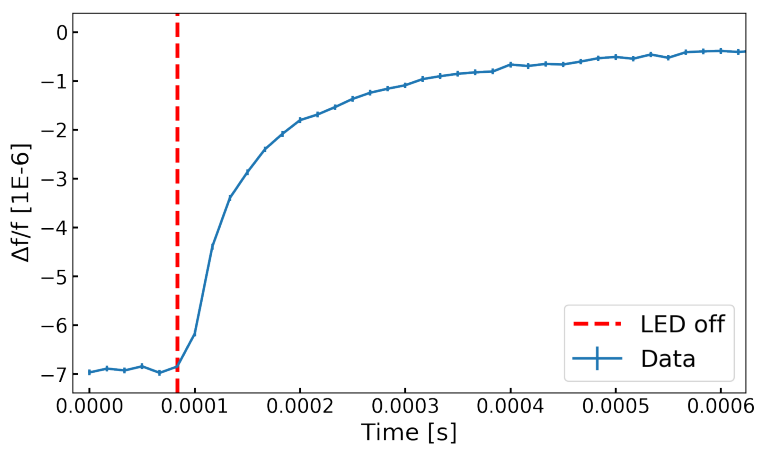

Figure 4. A pulse of a single resonator (blue points and line) at $100 \mathrm{mK}$ and the point in time at which the LED turns off (red dashed line). Notably, the decay does not begin immediately after the LED is turned off, but rather approximately two data points $(50 \mu \mathrm{s})$ later.

\section{REFERENCES}

[1] Glenn, J. et al., "The galaxy evolution probe: A concept for a mid and far-infrared space observatory," SPIE Proceedings (10698-20) (2018).

[2] Janssen, R. M. J. et al., "Performance of hybrid nbtin-al microwave kinetic inductance detectors as direct detectors for sub-millimeter astronomy," SPIE Proceedings (2014).

[3] Diener, P. et al., "Design and testing of kinetic inductance detectors made of titanium nitride," $J$ Low Temp Phys 167(305) (2012). 

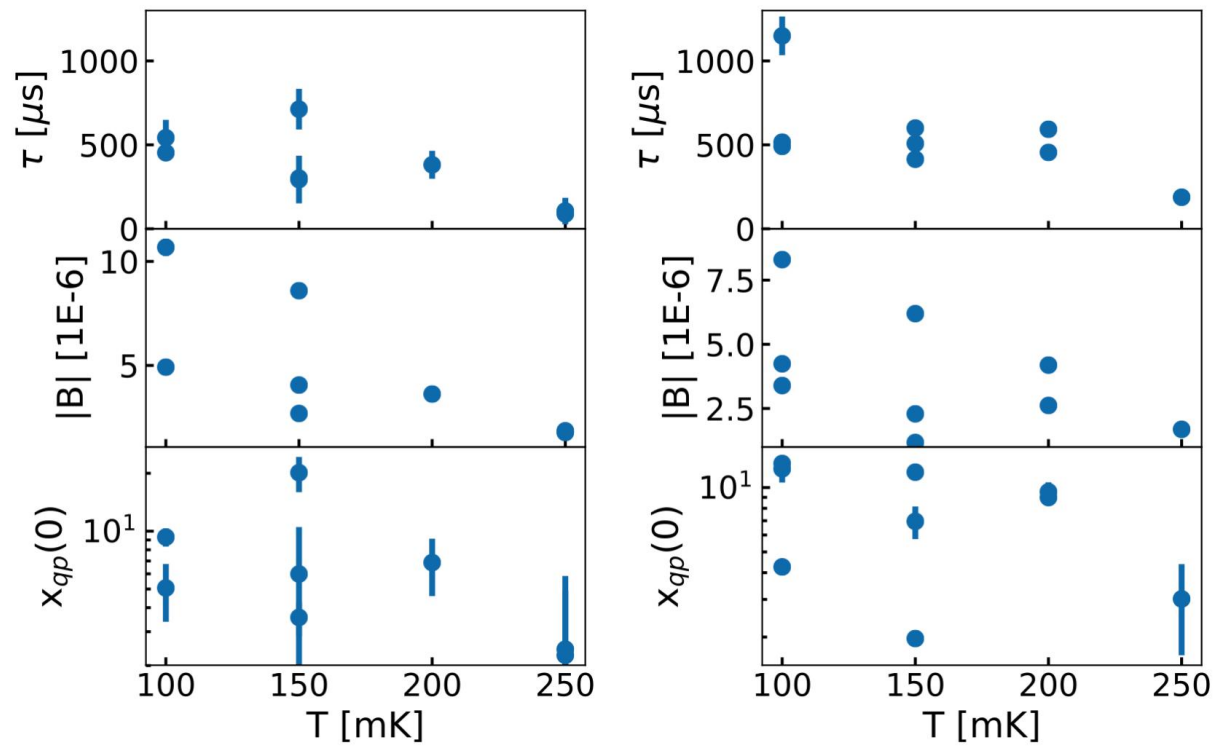

Figure 5. The fit parameters from Equation 9 as a function of temperature for two resonators on an aluminum device of thickness $40 \mathrm{~nm}$. Both resonators were driven around $3 \mathrm{~dB}$ below bifurcation with the same LED luminosity.

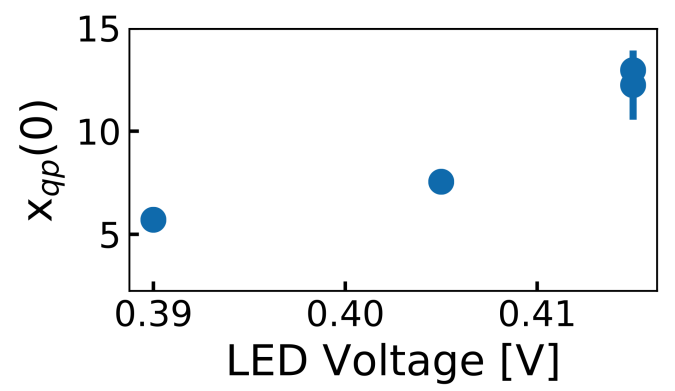

Figure 6. $\mathrm{x}_{q p}(0)$ as a function of LED driving voltage, a proxy for luminosity.

[4] Kenyon, M. et al., "Ultrasensitive transitionedge sensors (tess) for farir/submm spaceborne spectroscopy," AIR Conference Proceedings (2009).

[5] Barends, R. et al., "Quasiparticle relaxation in optically excited high-q superconducting resonators," Physical Review Letters 100(257002) (2008).

[6] de Visser, P. et al., "Number fluctuations of sparse quasiparticles in a superconductor," Physical Review Letters 106(167004) (2011).

[7] Glenn, J. et al., "Low-volume aluminum and aluminum/titanium nitride bilayer lumped-element kinetic inductance detectors for far-infrared astronomy," SPIE Proceedings (2016).

[8] Campbell, T. et al., "Dynamics of oxidation of aluminum nanoclusters using variable charge moleculardynamics simulations on parallel computers," Physical Review Letters 82(24) (1999).

[9] Fyhrie, A. et al., "Towards background-limited kinetic inductance detectors for a cryogenic far-infrared space telescope," J Low Temp Phys 184(3) (2016). 